\title{
Extension of Khan's Homotopy Transformation Method via Optimal Parameter for Differential Difference Equations
}

\author{
Mohamed S. Mohamed, ${ }^{1,2}$ Khaled A. Gepreel, ${ }^{1,3}$ Faisal A. Al-Malki, ${ }^{1}$ and Nouf Altalhi ${ }^{1}$ \\ ${ }^{1}$ Department of Mathematics, Faculty of Science, Taif University, Taif, Saudi Arabia \\ ${ }^{2}$ Department of Mathematics, Faculty of Science, Al Azhar University, Cairo, Egypt \\ ${ }^{3}$ Mathematics Department, Faculty of Science, Zagazig University, Zagazig, Egypt
}

Correspondence should be addressed to Mohamed S. Mohamed; m_s_mohamed2000@yahoo.com

Received 19 March 2014; Revised 11 September 2014; Accepted 12 September 2014; Published 23 November 2014

Academic Editor: Sheng Zhang

Copyright (c) 2014 Mohamed S. Mohamed et al. This is an open access article distributed under the Creative Commons Attribution License, which permits unrestricted use, distribution, and reproduction in any medium, provided the original work is properly cited.

\begin{abstract}
A new scheme, deduced from Khan's homotopy perturbation transform method (HPTM) (Khan, 2014; Khan and Wu, 2011) via optimal parameter, is presented for solving nonlinear differential difference equations. Simple but typical examples are given to illustrate the validity and great potential of Khan's homotopy perturbation transform method (HPTM) via optimal parameter in solving nonlinear differential difference equation. The numerical solutions show that the proposed method is very efficient and computationally attractive. It provides more realistic series solutions that converge very rapidly for nonlinear real physical problems. The results reveal that the method is very effective and simple. This method gives more reliable results as compared to other existing methods available in the literature. The numerical results demonstrate the validity and applicability of the method.
\end{abstract}

\section{Introduction}

Nonlinear differential difference equations (NDDEs) play a crucial role in many branches of applied physical sciences such as condensed matter physics, biophysics, atomic chains, molecular crystals, and discretization in solid-state and quantum physics. They also play an important role in numerical simulation of solution dynamics in high-energy physics because of their rich structures. Therefore, researchers have shown a wide interest in studying NDDEs since the original work of Fermi et al. [1] in the 1950s and [2, 3]. Contrary to difference equations that are being fully discretized, NDDEs are semidiscretized, with some (or all) of their space variables being discretized, while time is usually kept continuous. As far as we could verify, little work has been done to search for exact solutions of NDDEs. Hence, it would make sense to do more research on solving NDDEs. The approximate solution involves series of small parameters which poses difficulty since the majority of nonlinear problems have no small parameters at all. Although appropriate choices of small parameters sometime lead to ideal solutions in most cases, unsuitable choices lead to serious effects in the solutions. Therefore, an analytical method is welcome which does not require a small parameter in the equation modeling the phenomenon. The homotopy perturbation method (HPM) was first introduced by He [4]. The HPM was also used by many researchers to investigate various linear and nonlinear equations arising in physics and engineering. Many analytic approximate approaches for solving nonlinear differential equations have been proposed and the most outstanding one is the homotopy analysis method (HAM). In recent years, many authors have paid attention to studying the solutions of nonlinear partial differential equations and nonlinear differential difference equations by various methods [5-25].

The objective of the present paper is to extend the application of the HPTM to obtain an analytic approximate solution of the following nonlinear difference differential equations in mathematical physics:

(i) the general lattice equation [1-3]:

$$
\frac{d u_{n}}{d t}=\left(\alpha+\beta u_{n}+\gamma u_{n}^{2}\right)\left(u_{n+1}-u_{n-1}\right) ;
$$


(ii) the relativistic Toda lattice system [1-3]:

$$
\begin{gathered}
\frac{d u_{n}}{d t}-\left(1+\alpha u_{n}\right)\left(v_{n}-v_{n-1}\right)=0 \\
\frac{d v_{n}}{d t}-v_{n}\left(u_{n+1}-u_{n}+\alpha v_{n+1}-\alpha v_{n-1}\right)=0
\end{gathered}
$$

where $\alpha, \beta$, and $\gamma$ are arbitrary constants. The HPTM provides the solutions in terms of convergent series with easily computable components in a direct way without using linearization, perturbation, or restrictive assumptions. It is worth mentioning that the proposed approach is capable of reducing the volume of computational work compared to classical methods while still maintaining the high accuracy in the numerical result; the size reduction amounts to an improvement of the performance of the approach. The technique is based on the application of the Laplace transform via the homotopy method to solve nonlinear differential difference models. The main advantage of this problem is that we can accelerate the convergence rate, minimize iterative times, accordingly save the computation time, and evaluate the efficiency. Several examples are given to access the reliability of HPTM via optimal parameter.

\section{Description of the Homotopy Perturbation Transform Method}

We consider a general nonlinear, nonhomogenous partial differential equation

$$
L u(x, t)+R u(x, t)+N u(x, t)=f(x),
$$

where $L$ is a linear operator, $N$ is a nonlinear operator, and $f(x, t)$ is a source function. The initial conditions are also as

$$
u(x, 0)=g(x), \quad u_{t}(x, 0)=f(x) .
$$

Applying the Laplace transforms $\mathscr{L}$ on both sides, we get

$$
\mathscr{L}[\operatorname{Lu}(x, t)+R u(x, t)+N u(x, t)=f(x)]
$$
we get

Using the differentiation property of Laplace transform,

$$
\begin{gathered}
s^{2} \mathscr{L}[u]-s u(x, 0)-u_{t}(x, 0) \\
=\mathscr{L} f(x, t)-\mathscr{L} R u(x, t)-\mathscr{L} N u(x, t), \\
\mathscr{L} u(x, t)=\frac{h(x)}{s}+\frac{f(x)}{s^{2}}+\frac{1}{s^{2}} \mathscr{L} f(x, t) \\
-\frac{1}{s^{2}} \mathscr{L} R(x, t)-\frac{1}{s^{2}} \mathscr{L} \mathrm{Nu}(x, t) .
\end{gathered}
$$

Now we embed the homotopy in Laplace transform method. Hence we may write any equation in the form

$$
\begin{aligned}
& N\left[u_{i}(x, t)\right]=0, \quad i=1,2,3, \\
& N[\phi(x, t ; q)]= \frac{h(x)}{s}+\frac{f(x)}{s^{2}}+\frac{1}{s^{2}} \mathscr{L} f(x, t) \\
&-\frac{1}{s^{2}} \mathscr{L} R[\phi(x, t ; q)]-\frac{1}{s^{2}} \mathscr{L} N[\phi(x, t ; q)] \\
&-\mathscr{L}[\phi(x, t ; q)],
\end{aligned}
$$

where $\phi(x, t ; q)$ is a real function of $x, t$, and $q$. We construct a homotopy as

$$
(1-q) L\left[\phi(x, t ; q)-u_{0}(x, t)\right]=q \hbar H(x, t) N[u(x, t)],
$$

where $q \in[0,1]$ is an embedding parameter, $\hbar \neq 0$ is a nonzero auxiliary parameter, $H(x, t) \neq 0$ is a nonzero auxiliary function, $L$ is an auxiliary linear operator, $u_{0}(x, t)$ is an initial guess, and $\phi(x, t ; q)$ is an unknown function. It is important that one has great freedom to choose auxiliary things in homotopy deformation. Obviously, when $q=0$ and $q=1$, it holds

$$
\phi(x, t ; 0)=u_{0}(x, t), \quad \phi(x, t ; 1)=u(x, t) .
$$

The embedding parameter $q$ increases from 0 to 1 . Using Taylor's theorem, $\phi_{i}(x, t ; q)$ can be expanded in a power series of $q$ as follows:

$$
\phi(x, t ; q)=u_{0}(x, t)+\sum_{m=1}^{\infty} u_{m}(x, t) q^{m}
$$

where

$$
u_{m}(x, t)=\left.\frac{1}{m !} \frac{\partial^{m} \phi(x, t ; q)}{\partial q^{m}}\right|_{q=0} .
$$

If the auxiliary linear operator, the initial guess, the auxiliary parameter $\hbar$, and the auxiliary function are so properly chosen, series (10) converges at $q=1$; then we have

$$
u(x, t)=u_{0}(x, t)+\sum_{m=1}^{\infty} u_{m}(x, t)
$$

which must be one of the solutions of the original nonlinear equation. The governing equation can be deduced from zeroorder deformation equation (8).

Define the vector

$$
\vec{u}_{n}=\left\{u_{0}(x, t), u_{1}(x, t), \ldots, u_{n}(x, t)\right\} .
$$

Differentiating (8) $m$-times with respect to the embedding parameter $q$, then setting $q=0$, and finally dividing them by $m$ !, we obtain the $m$ th-order deformation equation:

$$
\mathscr{L}\left[u_{m}(x, t)-\chi_{m} u_{m-1}(x, t)\right]=\hbar H(x, t) R_{m}\left(\vec{u}_{m-1}\right) .
$$


Applying inverse Laplace transform we get

$$
u_{m}(x, t)=\chi_{m} u_{m-1}+\hbar \mathscr{L}^{-1}\left[H(x, t) R_{m}\left(\vec{u}_{m-1}\right)\right],
$$

where

$$
\begin{gathered}
R_{m}\left(\vec{u}_{m-1}\right)=\left.\frac{1}{(m-1) !} \frac{\partial^{m-1} N[\phi(x, t ; q)]}{\partial q^{m-1}}\right|_{q=0}, \\
\chi_{m}= \begin{cases}0, & m \leq 1, \\
1, & m>1 .\end{cases}
\end{gathered}
$$

$m$ th-order deformation equation (15) is a linear iteration problem and thus can be easily solved, especially by means of symbolic computation software such as Mathematica or Maple.

\section{Numerical Results}

To demonstrate the effectiveness of the HPTM algorithm discussed above [19-28], several examples of variational problems will be studied in this section.

Example 1. Consider the following nonlinear difference differential equation:

$$
\frac{d u_{n}}{d t}=\left(\alpha+\beta u_{n}+\gamma u_{n}^{2}\right)\left(u_{n-1}-u_{n+1}\right)
$$

with the initial condition

$$
u_{n}(0)=\frac{\sqrt{\beta^{2}-4 \alpha \gamma} \operatorname{sn}(\lambda, \mu) c n(\lambda n, \mu) d n(\lambda n, \mu)}{2 \gamma c n(\lambda, \mu) d n(\lambda, \mu) \operatorname{sn}(\lambda n, \mu)}-\frac{\beta}{2 \gamma}
$$

with the exact solution

$$
u_{n}(t)=\frac{\sqrt{\beta^{2}-4 \alpha \gamma} \operatorname{sn}(\lambda, m) c n(\zeta, m) d n(\zeta, m)}{2 \gamma c n(\lambda, \mu) d n(\lambda, \mu) \operatorname{sn}(\zeta, \mu)}-\frac{\beta}{2 \gamma},
$$

where $\operatorname{sn}(\lambda, \mu), c n(\lambda n, \mu)$, and $d n(\lambda n, \mu)$ are Jacobian functions and $\alpha, \beta, \gamma, \lambda$, and $\mu$ are arbitrary constants and

$$
\zeta=\lambda n-\frac{t\left(4 \alpha \gamma-\beta^{2}\right) s n(\lambda, \mu)}{2 \gamma c n(\lambda, \mu) d n(\lambda, \mu)} .
$$

To solve (17) by means of the transform method we consider the following linear equation:

$$
\mathscr{L}[\phi(x, t ; q)]=\frac{\partial \phi(x, t ; q)}{\partial t}
$$

with the property that

$$
\mathscr{L}\left[c_{1}\right]=0
$$

which implies that

$$
\mathscr{L}^{-1}(\cdot)=\int_{0}^{t}(\cdot) d t
$$

Taking Laplace transform of (17) with both sides subject to the initial condition, we get

$$
\begin{aligned}
& \mathscr{L}\left[u_{n}(n, t)\right] \\
& -\frac{1}{s}\left[\frac{\sqrt{\beta^{2}-4 \alpha \gamma \operatorname{sn}}(\lambda, \mu) c n(\lambda n, \mu) d n(\lambda n, \mu)}{2 \gamma c n(\lambda, \mu) d n(\lambda, \mu) \operatorname{sn}(\lambda n, \mu)}\right] \\
& -\frac{1}{s} \mathscr{L}\left[\left(\alpha+\beta u_{n}+\gamma u_{n}^{2}\right)\left(u_{n-1}-u_{n+1}\right)\right]=0 .
\end{aligned}
$$

We now define the nonlinear operator as

$$
\begin{aligned}
& N\left[\phi_{n}(t ; q)\right] \\
& =\mathscr{L}\left[\phi_{n}(t ; q)\right] \\
& -\frac{1}{s}\left[\frac{\sqrt{\beta^{2}-4 \alpha \gamma} \operatorname{sn}(\lambda, \mu) c n(\lambda n, \mu) d n(\lambda n, \mu)}{2 \gamma c n(\lambda, \mu) d n(\lambda, \mu) s n(\lambda n, \mu)}\right] \\
& -\frac{1}{s} \mathscr{L}\left[\left(\alpha+\beta \phi_{n}(t ; q)+\gamma \phi_{n}(t ; q)^{2}\right)\right. \\
& \left.\quad \times\left(\phi_{n-1}(t ; q)-\phi_{n+1}(t ; q)\right)\right]=0
\end{aligned}
$$

and then the $m$ th-order deformation equation is given by

$$
\mathscr{L}\left[u_{m}(n, t)-\chi_{m} u_{m-1}(n, t)\right]=\hbar H(n, t) R_{m}\left(\vec{u}_{m-1}\right) .
$$

Taking inverse Laplace transform of (26), we get

$$
u_{m}(n, t)=\chi_{m} u_{m-1}+\hbar \mathscr{L}^{-1}\left[H(n, t) R_{m}\left(\vec{u}_{m-1}\right)\right]
$$

where

$$
\begin{aligned}
R_{m}\left(\vec{u}_{m-1}\right) & \\
= & \mathscr{L}\left[u_{m}\right] \\
& -\frac{1}{s}\left[\frac{\sqrt{\beta^{2}-4 \alpha \gamma s n}(\lambda, \mu) c n(\lambda n, \mu) d n(\lambda n, \mu)}{2 \gamma c n(\lambda, \mu) d n(\lambda, \mu) \operatorname{sn}(\lambda n, \mu)}\right] \\
& \times\left(1-\chi_{m}\right) \\
& -\frac{1}{s} \mathscr{L}\left[\left(\alpha+\beta u_{m-1}(n, t)+\gamma u_{m-1}^{2}(n, t)\right)\right. \\
& \left.\quad \times\left(u_{m-1}(n-1, t)-u_{m-1}(n+1, t)\right)\right] .
\end{aligned}
$$

Let us take the initial approximation as

$u_{0}(n, t)=\frac{\sqrt{\beta^{2}-4 \alpha \gamma \sin }(\lambda, \mu) c n(\lambda n, \mu) d n(\lambda n, \mu)}{2 \gamma c n(\lambda, \mu) d n(\lambda, \mu) \operatorname{sn}(\lambda n, \mu)} ;$ 
the other components are given by

$$
\begin{aligned}
& u_{1}(n, t) \\
& =-h t(\alpha \\
& +\frac{\left(\beta^{2}-4 \alpha \gamma\right) c n[n \lambda, \mu]^{2} d n[n \lambda, \mu]^{2} \operatorname{sn}[\lambda, \mu]^{2}}{4 \gamma c n[\lambda, \mu]^{2} d n[\lambda, \mu]^{2} \operatorname{sn}[n \lambda, \mu]^{2}} \\
& \left.+\frac{\beta \sqrt{\beta^{2}-4 \alpha \gamma c n}[n \lambda, \mu] d n[n \lambda, \mu] \operatorname{sn}[\lambda, \mu]}{2 \gamma c n[\lambda, \mu] d n[\lambda, \mu] \operatorname{sn}[n \lambda, \mu]}\right) \\
& \times\left(\left(\left(\sqrt{\beta^{2}-4 \alpha \gamma} c n[(-1+n) \lambda, \mu]\right.\right.\right. \\
& \times d n[(-1+n) \lambda, \mu] \operatorname{sn}[\lambda, \mu]) \\
& \times(2 \gamma c n[\lambda, \mu] d n[\lambda, \mu] \\
& \left.\times s n[(-1+n) \lambda, \mu])^{-1}\right) \\
& -\left(\left(\sqrt{\beta^{2}-4 \alpha \gamma c n}[(1+n) \lambda, \mu]\right.\right. \\
& \times d n[(1+n) \lambda, \mu] \operatorname{sn}[\lambda, \mu]) \\
& \times(2 \gamma c n[\lambda, \mu] d n[\lambda, \mu] \\
& \left.\left.\times s n[(1+n) \lambda, \mu])^{-1}\right)\right) \\
& \text {... ; }
\end{aligned}
$$

other components of the approximate solution can be obtained in the same manner. Therefore, the approximate solution is

$$
u(n, t)=u_{0}(n, t)+u_{1}(n, t)+u_{2}(n, t)+\cdots
$$

We calculate the numerical results for different values of $n \in[-30,30] ; \alpha=0.2, \beta=0.5, \gamma=0.2, \lambda=0.3$, and $t=0.45$ are presented in Figures 1 and 2 . The comparison between HPTM via optimal parameter and the exact solution is performed in Figures 3 and 4. A very good agreement is achieved between the results obtained by the presented method HPTM via optimal parameter and the exact solution (Figure 6) for different values of $\mu$.

The Jacobi elliptic functions are generated into hyperbolic functions when $\mu \rightarrow 1$ as follows,

$$
\begin{gathered}
\operatorname{sn}(\xi) \longrightarrow \tanh (\xi), \quad c n(\xi) \longrightarrow \operatorname{sech}(\xi), \\
d n(\xi) \longrightarrow \operatorname{sech}(\xi),
\end{gathered}
$$

and into trigonometric functions when $\mu \rightarrow 0$ as follows:

$$
\begin{gathered}
\operatorname{sn}(\xi) \longrightarrow \sin (\xi), \quad c n(\xi) \longrightarrow \cos (\xi), \\
d n(\xi) \longrightarrow 1 .
\end{gathered}
$$

Example 2. Let us consider the following problem:

$$
\begin{gathered}
\frac{d}{d t} u_{n}-\left(1+\alpha u_{n}\right)\left(v_{n}-v_{n-1}\right)=0, \\
\frac{d}{d t} u_{n}-v_{n}\left(u_{n+1}-u_{n}+\alpha v_{n+1}-\alpha v_{n-1}\right)=0,
\end{gathered}
$$

with the initial condition

$$
\begin{gathered}
u_{0}(n, 0)=\frac{-\left(e^{\lambda}-1+\alpha c\right)}{\alpha\left(e^{\lambda}-1\right)}-\frac{c \beta}{\left(\beta+e^{n \lambda+\zeta}\right)}, \\
v_{0}(n, 0)=\frac{c}{\alpha\left(e^{\lambda}-1\right)}+\frac{c \beta}{\alpha\left(\beta+e^{n \lambda+\zeta}\right)},
\end{gathered}
$$

with the exact solution

$$
\begin{gathered}
u(n, t)=\frac{-\left(e^{\lambda}-1+\alpha c\right)}{\alpha\left(e^{\lambda}-1\right)}-\frac{c \beta}{\left(\beta+e^{c t+n \lambda+\zeta}\right)}, \\
v(n, t)=\frac{c}{\alpha\left(e^{\lambda}-1\right)}+\frac{c \beta}{\alpha\left(\beta+e^{c t+n \lambda+\zeta}\right)},
\end{gathered}
$$

where $\alpha, \beta, c, \lambda, n$, and $\zeta$ are arbitrary constants. Let

$$
\mathscr{L}[\phi(t ; q)]=\frac{\partial \phi(t ; q)}{\partial t}
$$

with the property that

$$
\mathscr{L}\left[c_{1}\right]=0
$$

which implies that

$$
\mathscr{L}^{-1}(\cdot)=\int_{0}^{t}(\cdot) d t .
$$

Taking Laplace transform of (34) with both sides subject to the initial condition, we get

$$
\begin{gathered}
\mathscr{L}\left[u_{n}(x, t)\right]-\frac{1}{s}\left[\frac{-\left(e^{\lambda}-1+\alpha c\right)}{\alpha\left(e^{d}-1\right)}-\frac{c \beta}{\left(\beta+e^{n \lambda+\zeta}\right)}\right] \\
\quad-\frac{1}{s} \mathscr{L}\left[\left(1+\alpha u_{n}\right)\left(v_{n}-v_{n-1}\right)\right]=0, \\
\mathscr{L}\left[v_{n}(x, t)\right]-\frac{1}{s}\left[\frac{c}{\alpha\left(e^{d}-1\right)}+\frac{c \beta}{\alpha\left(\beta+e^{n \lambda+\zeta}\right)}\right] \\
\quad-\frac{1}{s} \mathscr{L}\left[v_{n}\left(u_{n+1}-u_{n}+\alpha v_{n+1}-\alpha v_{n-1}\right)\right]=0 .
\end{gathered}
$$




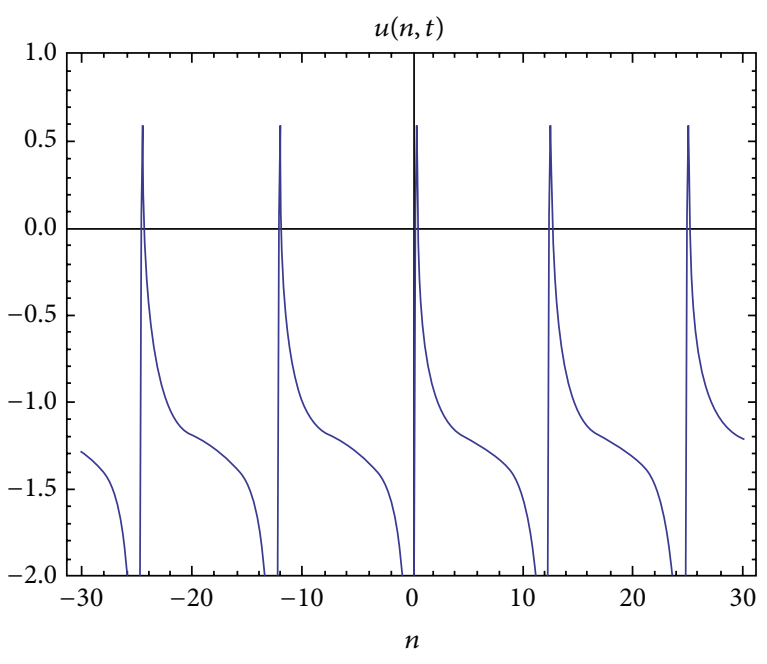

(a)

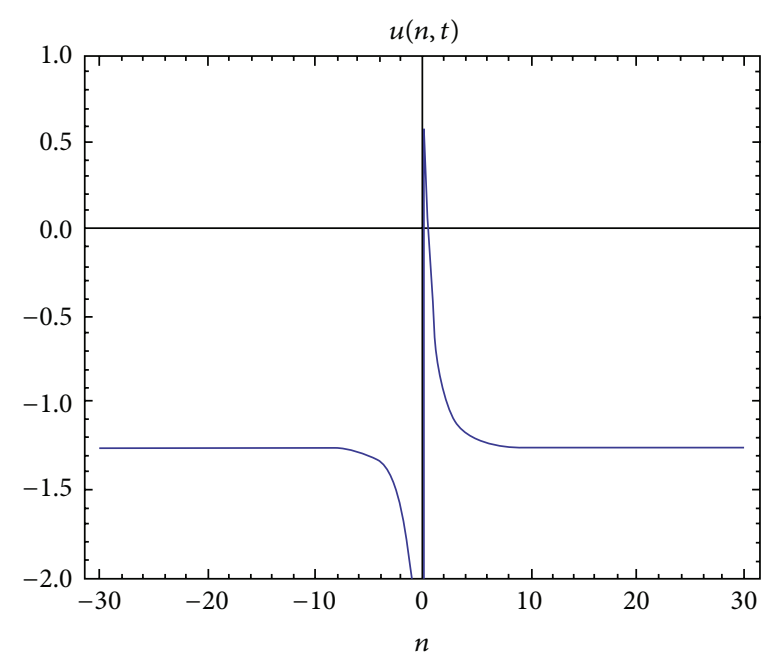

(b)

FIGURE 1: The 2nd-order approximate solution (31), respectively, when $\mu=0.5$ and 1 at $\left.h\right|_{\text {optimal }}=-0.975$.

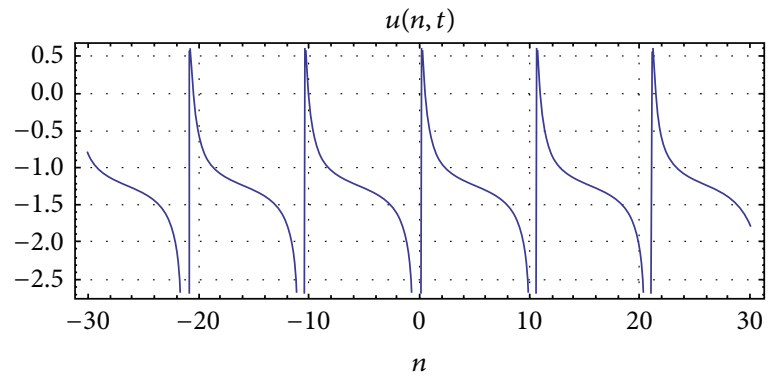

FIgURE 2: The 2nd-order approximate solution (31) when $\mu=0$.

We now define the nonlinear operator as

$$
\begin{aligned}
N_{1}\left[\phi_{n}(t ; q)\right]= & \mathscr{L}\left[\phi_{n}(t ; q)\right] \\
- & -\frac{1}{s}\left[\frac{-\left(e^{\lambda}-1+\alpha c\right)}{\alpha\left(e^{d}-1\right)}-\frac{c \beta}{\left(\beta+e^{n \lambda+\zeta}\right)}\right] \\
- & \frac{1}{s} \mathscr{L}\left[\left(1+\alpha \phi_{n}(t ; q)\right)\right. \\
& \left.\quad \times\left(\psi_{n}(t ; q)-\psi_{n-1}(t ; q)\right)\right]=0,
\end{aligned}
$$$$
N_{2}\left[\psi_{n}(t ; q)\right]=\mathscr{L}\left[\psi_{n}(t ; q)\right]
$$

$$
\begin{array}{r}
-\frac{1}{s}\left[\frac{c}{\alpha\left(e^{d}-1\right)}+\frac{c \beta}{\alpha\left(\beta+e^{n \lambda+\zeta}\right)}\right] \\
-\frac{1}{s} \mathscr{L}_{2}\left[\psi_{n}(t ; q)\right. \\
\times\left(\phi_{n+1}(t ; q)-\phi_{n}(t ; q)\right. \\
+\alpha \psi_{n-1}(t ; q) \\
\left.\left.-\alpha \psi_{n-1}(t ; q)\right)\right]=0
\end{array}
$$

and then the $m$ th-order deformation equation is given by

$$
\begin{aligned}
& \mathscr{L}\left[u_{m}(n, t)-\chi_{m} u_{m-1}(t, n)\right]=\hbar_{1} H_{1}(n, t) R_{1 m}\left(\vec{u}_{m-1}\right), \\
& \mathscr{L}\left[v_{m}(n, t)-\chi_{m} v_{m-1}(t, n)\right]=\hbar_{2} H_{2}(n, t) R_{2 m}\left(\vec{v}_{m-1}\right) .
\end{aligned}
$$

Taking inverse Laplace transform of (42), we get

$$
\begin{aligned}
& u_{m}(n, t)=\chi_{m} u_{m-1}+\hbar_{1} \mathscr{L}^{-1}\left[H_{1}(n, t) R_{1 m}\left(\vec{u}_{m-1}\right)\right], \\
& v_{m}(n, t)=\chi_{m} v_{m-1}+\hbar_{2} \mathscr{L}^{-1}\left[H_{2}(n, t) R_{2 m}\left(\vec{v}_{m-1}\right)\right],
\end{aligned}
$$

where

$$
\begin{aligned}
R_{1 m}\left(\vec{u}_{m-1}\right)= & \mathscr{L}\left[u_{m-1}\right] \\
& -\frac{1}{s}\left[\frac{-\left(e^{\lambda}-1+\alpha c\right)}{\alpha\left(e^{d}-1\right)}-\frac{c \beta}{\left(\beta+e^{n \lambda+\zeta}\right)}\right] \\
& \times\left(1-\chi_{m}\right) \\
& -\frac{1}{s} \mathscr{L}\left[\left(1+\alpha u_{m-1}(n, t)\right)\right. \\
& \left.\quad \times\left(v_{m-1}(n, t)-v_{m-1}(n-1, t)\right)\right],
\end{aligned}
$$




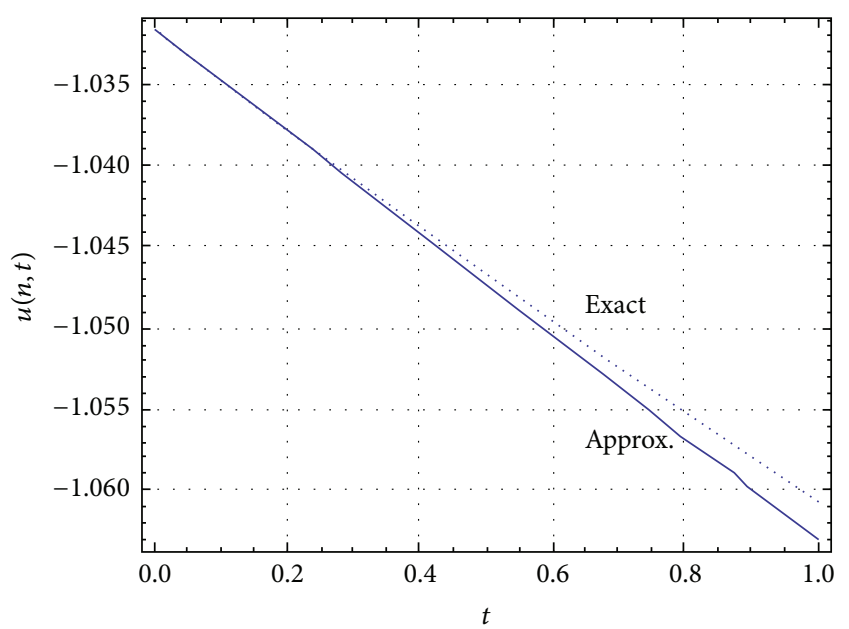

(a)

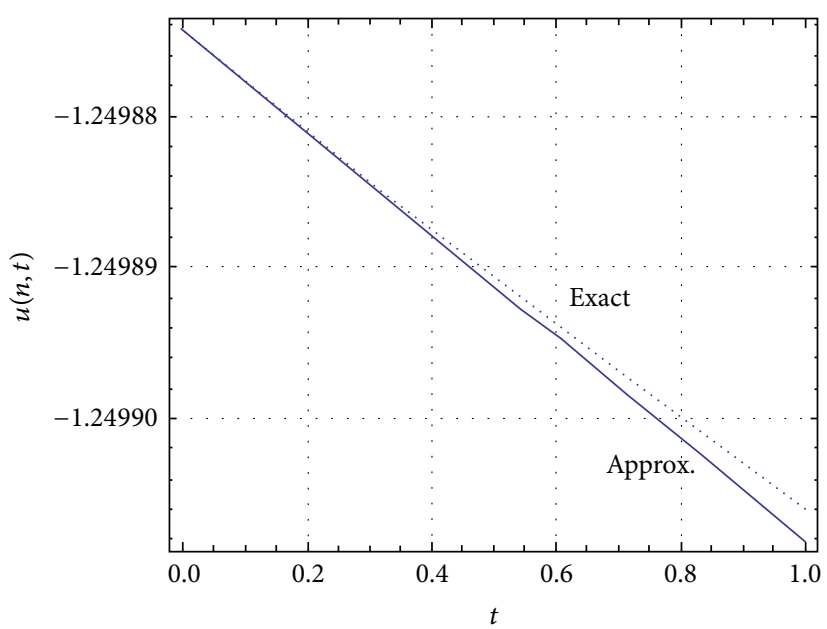

(b)

FIGURE 3: The behavior of the approximate solution using HPTM via optimal parameter when $\mu=0.5$ and 1 .

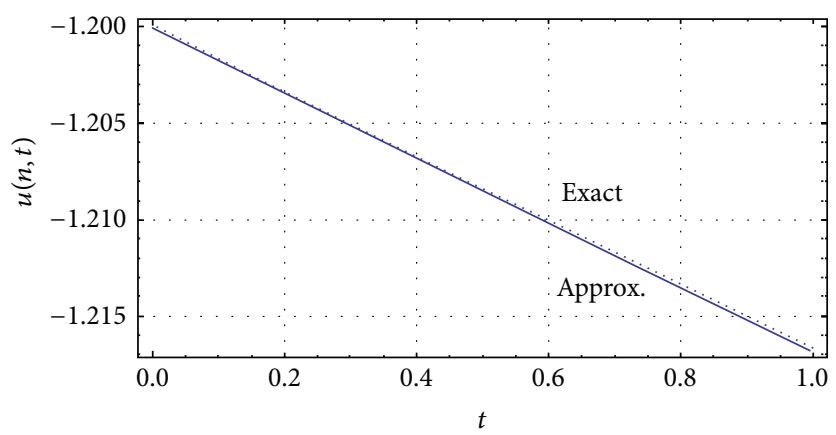

FIGURE 4: The behavior of the approximate solution using HPTM via optimal parameter when $\mu=0$.

$$
\begin{aligned}
& R_{2 m}\left(\vec{v}_{m-1}\right)= \mathscr{L}\left[v_{m-1}\right] \\
&-\frac{1}{s}\left[\frac{c}{\alpha\left(e^{d}-1\right)}+\frac{c \beta}{\alpha\left(\beta+e^{n \lambda+\zeta}\right)}\right]\left(1-\chi_{m}\right) \\
&-\frac{1}{s} \mathscr{L}\left[v_{m-1}(n, t)\right. \\
& \quad \times\left(u_{m-1}(n+1, t)-u_{m-1}(n, t)\right. \\
& \quad+\alpha v_{m-1}(n+1, t) \\
&\left.\left.\quad-\alpha v_{m-1}(n-1, t)\right)\right]
\end{aligned}
$$

the other components (Figure 5) are given by

$$
\begin{aligned}
u_{1}(n, t)= & -\frac{\beta c}{e^{\zeta+n \lambda}+\beta}-\frac{-1+e^{\lambda}+\alpha c}{\left(-1+e^{d}\right) \alpha} \\
& -h t\left(-\frac{\beta c}{\alpha\left(e^{\zeta+(-1+n) \lambda}+\beta\right)}+\frac{\beta c}{\alpha\left(e^{\zeta+n \lambda}+\beta\right)}\right) \\
& \times\left(1+\alpha\left(-\frac{\beta c}{e^{\zeta+n \lambda}+\beta}-\frac{-1+e^{\lambda}+\alpha c}{\left(-1+e^{d}\right) \alpha}\right)\right),
\end{aligned}
$$

where $h_{1}=h_{2}=-1$ and $H_{1}(n, t)=H_{2}(n, t)=1$.

Let us take the initial approximation as

$$
v_{1}(n, t)=\frac{c}{\left(-1+e^{d}\right) \alpha}+\frac{\beta c}{\alpha\left(e^{\zeta+n \lambda}+\beta\right)}
$$

$$
\begin{gathered}
u_{0}(n, 0)=\frac{-\left(e^{\lambda}-1+\alpha c\right)}{\alpha\left(e^{\lambda}-1\right)}-\frac{c \beta}{\left(\beta+e^{n \lambda+\zeta}\right)}, \\
v_{0}(n, 0)=\frac{c}{\alpha\left(e^{\lambda}-1\right)}+\frac{c \beta}{\alpha\left(\beta+e^{n \lambda+\zeta}\right)}
\end{gathered}
$$

$$
\begin{aligned}
& -h t\left(\frac{c}{\left(-1+e^{d}\right) \alpha}+\frac{\beta c}{\alpha\left(e^{\zeta+n \lambda}+\beta\right)}\right) \\
& \times\left(\frac{\beta c}{e^{\zeta+n \lambda}+\beta}-\frac{\beta c}{e^{\zeta+(1+n) \lambda}+\beta}\right. \\
& \quad-\alpha\left(\frac{c}{\left(-1+e^{d}\right) \alpha}+\frac{\beta c}{\alpha\left(e^{\zeta+(-1+n) \lambda}+\beta\right)}\right)
\end{aligned}
$$




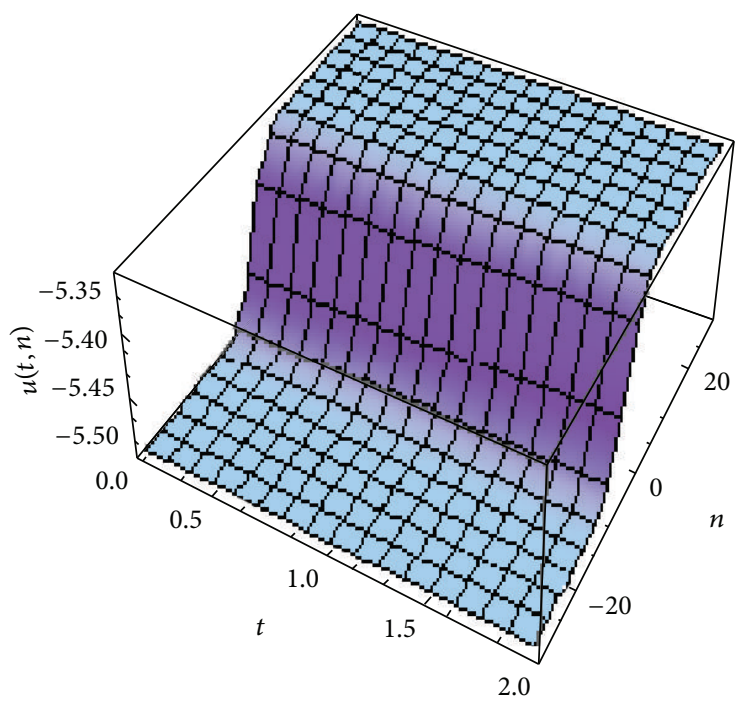

(a)

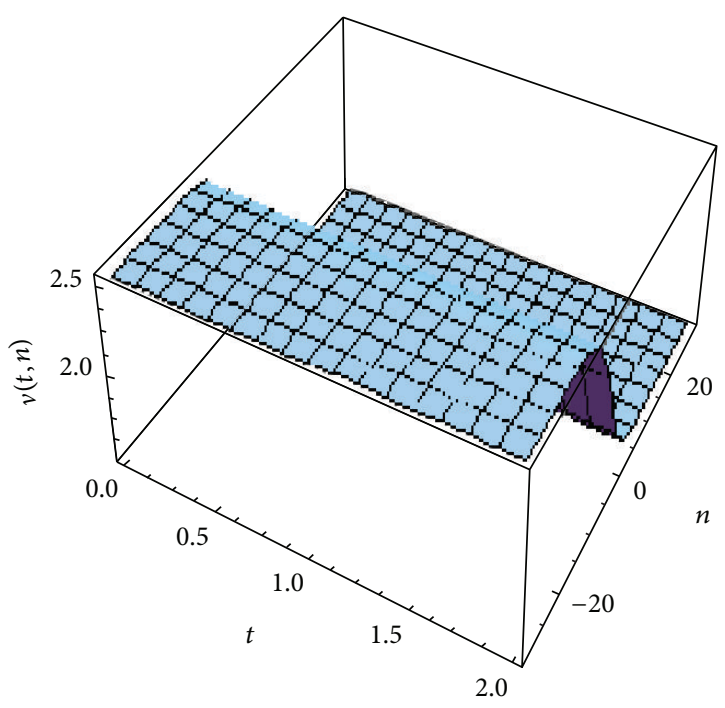

(b)

FIGURE 5: The 4th-order approximate solution (46) and (47), respectively, at $\left.h\right|_{\text {optimal }}=-0.993$.

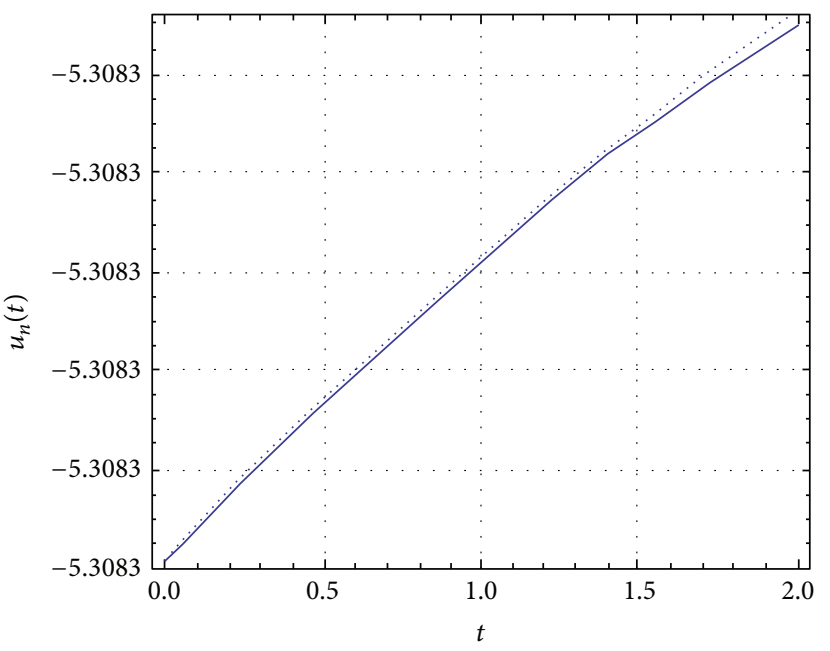

(a)

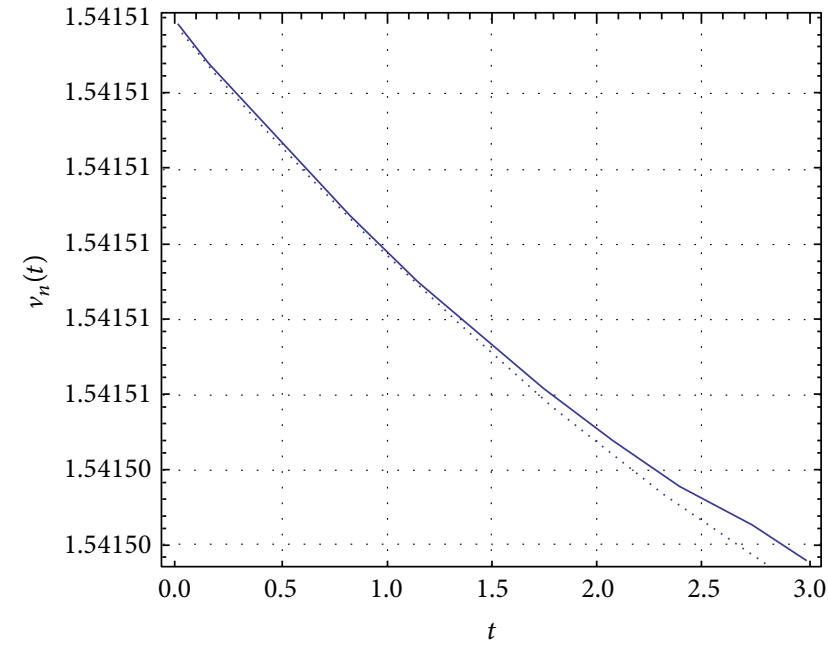

(b)

Figure 6: The behavior of the approximate solution using HPTM via optimal parameter and the exact solution (36).

$$
\left.+\alpha\left(\frac{c}{\left(-1+e^{d}\right) \alpha}+\frac{\beta c}{\alpha\left(e^{\zeta+(1+n) \lambda}+\beta\right)}\right)\right)
$$

\section{Conclusions}

In this paper, the HPTM via optimal parameter has been successfully applied for solving discontinued problems arising in nanotechnology. The result shows that the HPTM via optimal parameter is a powerful and efficient technique in finding exact and approximate solutions for nonlinear differential equations. Also, it can be observed that there is good agreement between the results obtained using the present method and the exact solution. The HPTM via optimal parameter requires less computational work compared to other analytical methods. In conclusion, the HPTM via optimal parameter may be considered a nice refinement in existing numerical techniques and may find wide applications. It is worth mentioning that the method is capable of reducing the volume of the computational work as compared to the classical methods while still maintaining high accuracy of the numerical result, and the size reduction amounts to the improvement of performance of the approach. The method overcomes the difficulty encountered in other methods because it is efficient. Comparisons have been made among the exact solutions, 
the Adomian decomposition method, the variational iteration method, and the HPM. We obtained the best results by using HPTM and we confirm that engineers can use these results for any application especially in textile engineering.

\section{Conflict of Interests}

The authors declare that there is no conflict of interests regarding the publication of this paper.

\section{References}

[1] E. Fermi, J. Pasta, and S. Ulam, Collected Papers of Enrico Fermi II, University of Chicago Press, Chicago, Ill, USA, 1965.

[2] K. A. Gepreel, T. A. Nofal, and F. M. Alotaibi, "Exact solutions for nonlinear differential difference equations in mathematical physics," Abstract and Applied Analysis, vol. 2013, Article ID 756896, 10 pages, 2013.

[3] K. A. Gepreel, T. A. Nofal, and A. A. Al-Thobaiti, "The modified rational Jacobi elliptic functions method for nonlinear differential difference equations," Journal of Applied Mathematics, vol. 2012, Article ID 427479, 30 pages, 2012.

[4] J.-H. He, "A coupling method of a homotopy technique and a perturbation technique for non-linear problems," International Journal of Non-Linear Mechanics, vol. 35, no. 1, pp. 37-43, 2000.

[5] J.-H. He, "Application of homotopy perturbation method to nonlinear wave equations," Chaos, Solitons and Fractals, vol. 26, no. 3, pp. 695-700, 2005.

[6] D. D. Ganji and A. Sadighi, "Application of He's homotopyperturbation method to nonlinear coupled systems of reactiondiffusion equations," International Journal of Nonlinear Sciences and Numerical Simulation, vol. 7, no. 4, pp. 411-418, 2006.

[7] J.-H. He, "Homotopy perturbation method for solving boundary value problems," Physics Letters A, vol. 350, no. 1-2, pp. 8788, 2006.

[8] M. S. Mohamed, F. AL-Malki, and N. Altalhi, "Analytic and approximate solutions of time and space fractional nonlinear cubic equations via Laplace transform," Jokull Journal, vol. 64, no. 6, pp. 490-503, 2014.

[9] K. A. Gepreel and M. S. Mohamed, "An optimal homotopy analysis method nonlinear fractional differential equation," Journal of Advanced Research in Dynamical and Control Systems, vol. 6, no. 1, pp. 1-10, 2014.

[10] A.-M. Wazwaz, "The variational iteration method: a powerful scheme for handling linear and nonlinear diffusion equations," Computers \& Mathematics with Applications, vol. 54, no. 7-8, pp. 933-939, 2007.

[11] Y. Khan and F. Austin, "Application of the Laplace decomposition method to nonlinear homogeneous and non-homogeneous advection equations," Zeitschrift für Naturforschung A, vol. 65, pp. 1-5, 2010.

[12] M. Madani, M. Fathizadeh, Y. Khan, and A. Yildirim, "On the coupling of the homotopy perturbation method and Laplace transformation," Mathematical and Computer Modelling, vol. 53, no. 9-10, pp. 1937-1945, 2011.

[13] Y. Khan and Q. Wu, "Homotopy perturbation transform method for nonlinear equations using He's polynomials," Computers \& Mathematics with Applications, vol. 61, no. 8, pp. 19631967, 2011.

[14] F. Abidi and K. Omrani, "The homotopy analysis method for solving the Fornberg-Whitham equation and comparison with
Adomian's decomposition method," Computers \& Mathematics with Applications, vol. 59, no. 8, pp. 2743-2750, 2010.

[15] M. M. Khader, "Numerical solution for discontinued problems arising in nanotechnology using HAM," Journal of Nanotechnology \& Advanced Materials, vol. 1, no. 1, pp. 59-67, 2013.

[16] A. Yıldırım, "He’s homotopy perturbation method for nonlinear differential-difference equations," International Journal of Computer Mathematics, vol. 87, no. 5, pp. 992-996, 2010.

[17] S. Jagdev, K. Devendra, and K. Sunil, "A reliable algorithm for solving discontinued problems arising in nanotechnology," Scientia Iranica, vol. 20, no. 3, pp. 1059-1062, 2013.

[18] Z. Obidat and S. Momani, "The homotopy analysis method for solving discontinued problems arising in nanotechnology," World Academy of Science, Engineering and Technology, vol. 52, pp. 891-894, 2011.

[19] H. Hosseinzadeh, H. Jafari, and M. Roohani, "Application of Laplace decomposition method for solving Klein-Gordon equation," World Applied Sciences, vol. 8, no. 7, pp. 809-813, 2010.

[20] N. Faraza, Y. Khana, and F. Austinb, "An alternative approach to differential-difference equations using the variational iteration method," Zeitschriftfuer Naturforschung, vol. 65a, pp. 1055-1059, 2010.

[21] Y. Khan, H. Vzquez-Leal, and N. Faraz, "An auxiliary parameter method using Adomian polynomials and Laplace transformation for nonlinear differential equations," Applied Mathematical Modelling, vol. 37, no. 5, pp. 2702-2708, 2013.

[22] Y. Khan and Q. Wu, "Homotopy perturbation transform method for nonlinear equations using He's polynomials," Computers \& Mathematics with Applications, vol. 61, no. 8, pp. 19631967, 2011.

[23] Y. Khan, M. Madani, A. Yildirim, M. A. Abdou, and N. Faraz, "A new approach to Van der Pol's oscillator problem," Zeitschrift für Naturforschung A, vol. 66, pp. 620-624, 2011.

[24] Y. Khan, H. Vazquez-Leal, and L. Hernandez-Martínez, "Removal of noise oscillation term appearing in the nonlinear equation solution," Journal of Applied Mathematics, vol. 2012, Article ID 387365, 9 pages, 2012.

[25] K. A. Gepreel and M. S. Mohamed, "Improved $\left(G^{\prime} / G\right)$ expansion method for solving nonlinear difference differential equations," Journal of Computational and Theoretical Nanoscience. In press.

[26] Y. Khan, "An algorithm for solving nonlinear differentialdifference models," Computational Mathematics and Modeling, vol. 25, no. 1, pp. 115-123, 2014.

[27] K. A. Gepreel, "Jacobi elliptic numerical solutions for the time fractional Boussinesq equations," Journal of Partial Differential Equations, vol. 27, no. 3, pp. 189-199, 2014.

[28] K. A. Gepreel, "Exact solutions for nonlinear Toda Lattice difference differential equation," World Applied Sciences Journal, vol. 27, pp. 1792-1797, 2013. 


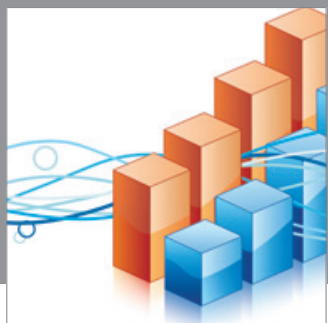

Advances in

Operations Research

mansans

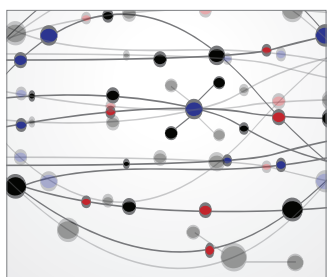

The Scientific World Journal
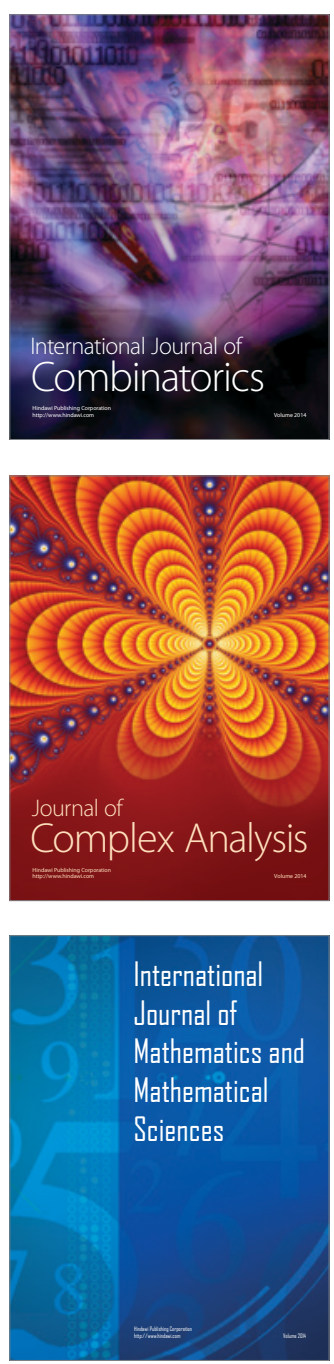
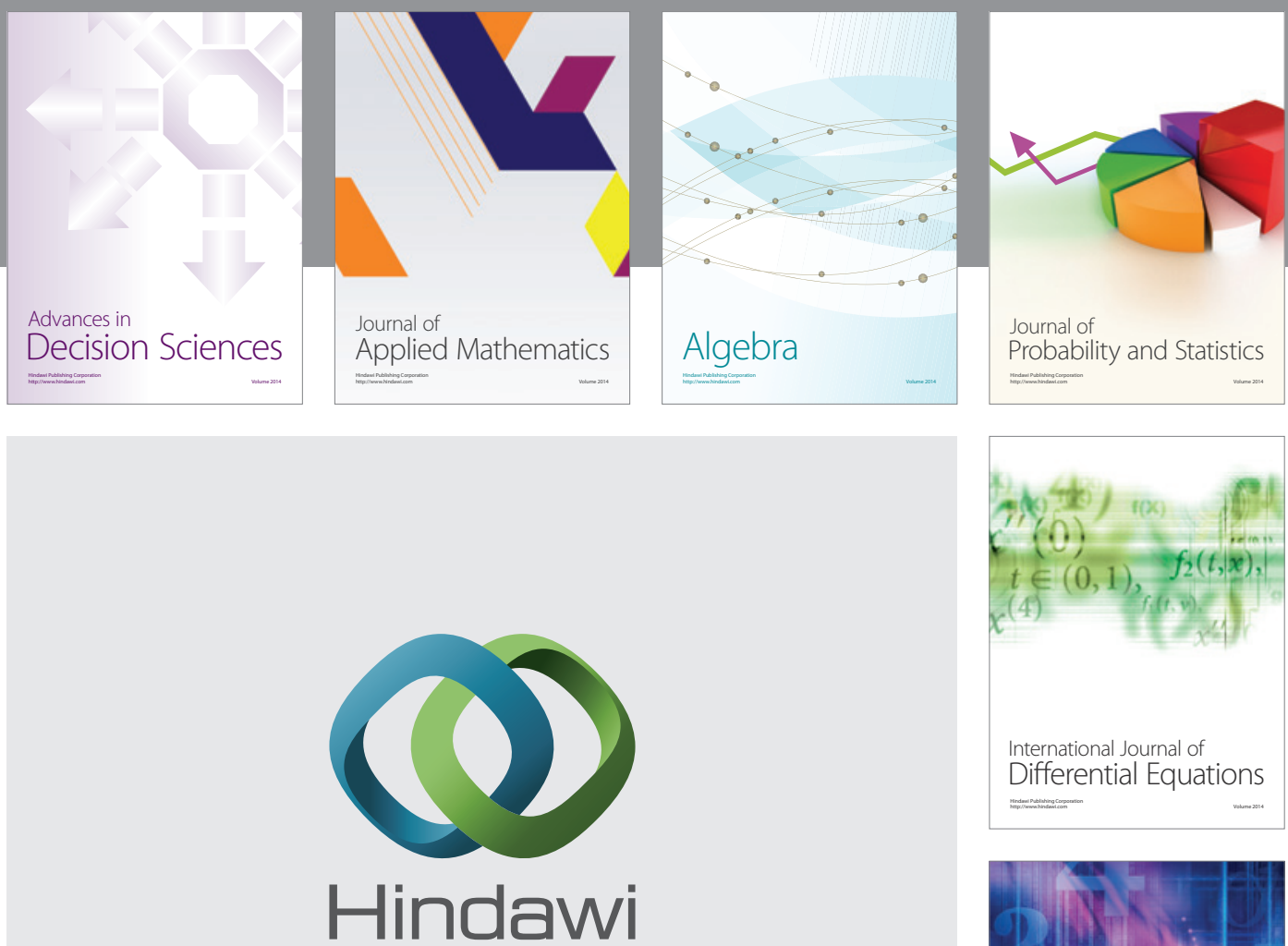

Submit your manuscripts at http://www.hindawi.com
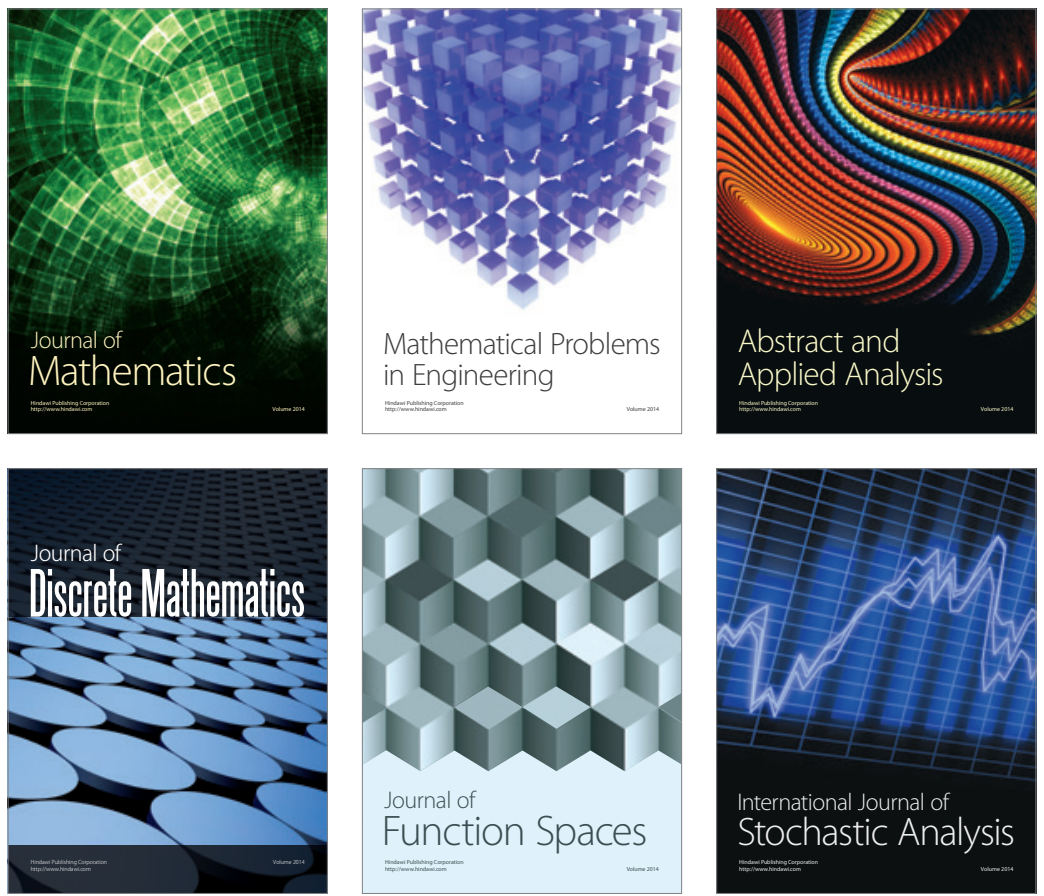

Journal of

Function Spaces

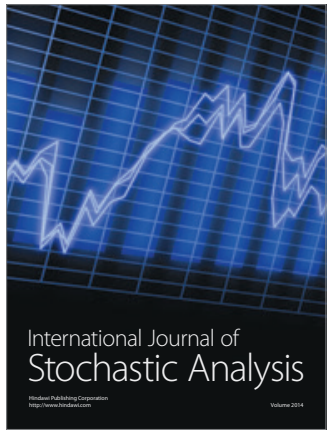

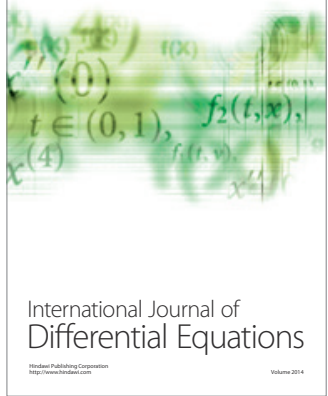
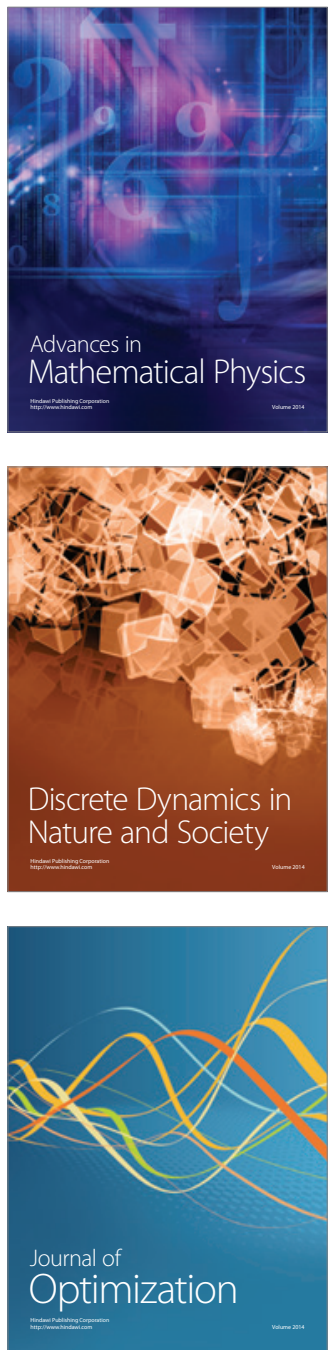